\title{
Is a binary fraction-age relation responsible for the lack of EHB binaries in globular clusters? ${ }^{\star}$
}

\author{
C. Moni Bidin ${ }^{1}$, M. Catelan ${ }^{2}$, and M. Altmann ${ }^{1,3}$ \\ 1 Departamento de Astronomía, Universidad de Chile, Casilla 36-D, Santiago, Chile \\ e-mail: mbidin@das.uchile.cl \\ 2 Pontificia Universidad Católica de Chile, Departamento de Astronomía y Astrofísica, Av. Vicuña Mackenna 4860, $782-0436$ Macul, \\ Santiago, Chile \\ 3 University of Heidelberg, Centre for Astronomy, Mönchhofstr. 12-14, 69120 Heidelberg, Germany
}

Received 2 October 2007 / Accepted 28 January 2008

\section{ABSTRACT}

\begin{abstract}
Context. The recently-discovered lack of close binaries, among extreme horizontal branch (EHB) stars in Galactic globular clusters, has thus far constituted a major puzzle, in view of the fact that blue subdwarf stars - the field counterparts of cluster EHB stars - are well-known to present a high binary fraction.

Aims. In this Letter, we provide new results that confirm the lack of close EHB binaries in globular clusters, and present a first scenario to explain the difference between field and cluster EHB stars.

Methods. First, in order to confirm that the lack of EHB binaries in globular clusters is a statistically robust result, we undertook a new analysis of 145 horizontal branch stars in NGC 6752, out of which forty-one belong to the EHB. To search for radial-velocity variations as a function of time, we repeated high-resolution $(R=18500)$ spectroscopy of all stars, four times during a single night of observations.

Results. We detected a single, hot $(25000 \mathrm{~K})$, radial-velocity variable star as a close-binary candidate. From these results, we estimate an upper-limit for the close (period $P \leq 5$ day) binary fraction $f$ among NGC 6752 EHB stars of 16\% (95\% confidence level), with the most probable value being $f=4 \%$. Thus our results clearly confirm the lack of close binaries among the hot HB stars in this cluster.

Conclusions. We suggest that the confirmed discrepancy between the binary fractions for field and cluster EHB stars is the consequence of an $f$-age relation, with close binaries being more likely in the case of younger systems. We analyze theoretical and observational results available in the literature, which support this scenario. If so, an age difference between the EHB progenitors in the field and in clusters, the former being younger (on average) by up to several Gyr, would naturally account for the startling differences in binary fraction between the two populations.
\end{abstract}

Key words. stars: horizontal-branch - binaries: close - binaries: spectroscopic - stars: subdwarfs globular clusters: individual: NGC 6752

\section{Introduction}

The presence of a large population of binaries among field B-type subdwarf (sdB) stars, also referred to as Extreme Horizontal Branch (EHB) stars, is well-established in the literature (Ferguson et al. 1984; Allard et al. 1994; Ulla \& Thejll 1998; Aznar Cuadrado \& Jeffery 2001; Maxted et al. 2001; Williams et al. 2001; Reed \& Stiening 2004; Napiwotzki et al. 2004). The measurement of binary fraction varies from one survey to another, probably because of the different survey selection effects. It is generally agreed however, that the binary fraction must be large. Moreover, extensive analysis of orbital parameters (Moran et al. 1999; Saffer et al. 1998; Heber et al. 2002; Morales-Rueda et al. 2004, 2006) reveal that close binaries ( $P \leq 10 \mathrm{~d})$ comprise a major fraction of the field EHB stars, with short-period systems - including either a degenerate or a low-mass main sequence (MS) companion - constituting about half of the entire field $\mathrm{sdB}$ population. Indeed, theoretical results indicate that these stars can be naturally explained within the context of

^ Based on observations with the ESO Very Large Telescope at Paranal Observatory, Chile (proposal ID 075.D-0492(A)). binary star evolution (Han et al. 2002, 2003). Moreover, EHB stars of binary origin may also account for the UV flux excess ("UV upturn") observed in elliptical galaxies (Han et al. 2007), although it should be noted that single-star scenarios have also been suggested (see Catelan 2007, for a recent review). One way or another, the link between field sdB stars and binary systems is very well-established, both on observational and theoretical grounds, and close, short-period systems are the most frequently found amongst them.

It therefore came as a great surprise that the first radialvelocity (RV) surveys among EHB stars in globular clusters (GCs) revealed a remarkable lack of close binary systems (see Moni Bidin et al. 2007a, for a review).

In this Letter we present, in light of new observational results, a first scenario to account for this discrepancy.

\section{Observations and measurements}

High-resolution spectra $(R=18500)$ centered on $\mathrm{H}_{\beta}(4700-$ $4970 \AA$ ) were collected with 3600s exposures on June 29, 2005 at VLT-UT2, with the FLAMES-GIRAFFE optical spectrograph 


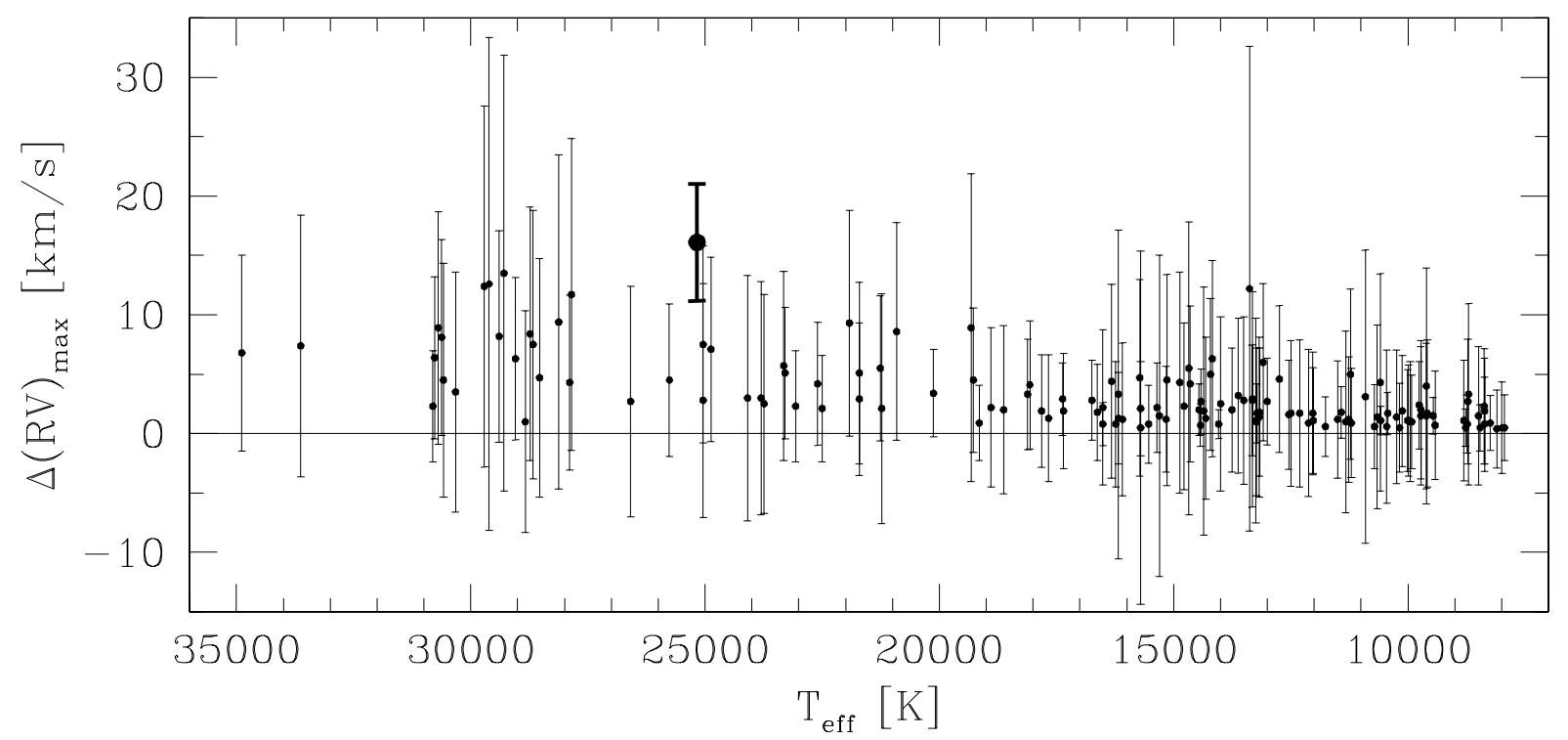

Fig. 1. Results of our $R V$ variability search. The maximum $R V$ variation $\left(R V_{\max }-R V_{\min }\right)$ observed for each star is plotted as a function of the effective temperature. For the sake of clarity, we plot only the $3 \sigma$ error bars, which indicate the statistical significance of the observed variations. The data for the only star with a clearly-detected RV variation, is plotted using a larger, solid circle symbol, and thicker lines to indicate its errorbar.

Table 1. Time elapsed (in hours) between the start of each exposure and the first one, for each fiber configuration (Medusa 1 and Medusa 2).

\begin{tabular}{c|cc}
\hline \hline Frame & \multicolumn{2}{|c}{$\begin{array}{c}\text { Medusa1 } \\
\text { hours }\end{array}$} \\
\hline 1 & 0.00 & 0.00 \\
2 & 1.03 & 4.73 \\
3 & 4.75 & 5.77 \\
4 & 5.78 & 9.13 \\
\hline
\end{tabular}

in MEDUSA configuration (setup HR7A). $169 \mathrm{HB}$ stars were selected from the Momany et al. (2002) photometry, spanning the whole temperature range along the $\mathrm{HB}$, out of which 54 were EHB stars (i.e., with $T_{\text {eff }} \geq 20000 \mathrm{~K}$ ). When performing the target selection, we ensured that each individual candidate was sufficiently isolated that no other bright star fell within the fiber, extending observations as far as possible toward the cluster center. Selected targets were divided into two fiber configurations. In Table 1, we indicate the time at the start of each exposure, in hours from the first spectrum. To confirm that our targets were true cluster HB stars, we verified that absolute radial velocities are consistent with cluster membership, and we inspected all spectra by eye. We excluded six, cool-star targets $\left(T_{\text {eff }} \approx 6500 \mathrm{~K}\right)$ from our analysis, because their cluster membership was doubtful.

All data reduction steps were completed using ESO's GIRAFFE pipeline. Wavelength calibration was performed using lamp fiber data acquired simultaneously during the observations: according to the FLAMES manual the calibration error is about $0.15 \mathrm{~km} \mathrm{~s}^{-1}$.

RV's were measured using the cross-correlation (CC) technique (Tonry \& Davis 1979), cross-correlating each spectrum with synthetic templates of appropriate temperature and gravity from the library of Munari et al. (2005). During CC we used only $\mathrm{H}_{\beta}$ with full wings, neglecting all weaker lines that were hardly visible due to noise, and highly variable from star to star. Some tests carried out prior to the actual measurements revealed that, with this limitation, the adopted metallicity of the template had a negligible impact on the results. Errors in the measured RV variations were calculated as the quadratic sum of the errors for the individual measurements.

More details about the observations, data reduction and measurements will be provided in a forthcoming paper (Moni Bidin et al., in preparation).

\section{Results}

We were unable to acquire observations for twelve stars, due to for example, light contamination by nearby fibers, too low signal-to-noise ratio, or the star being badly-centered in the fiber. Moreover, for six of the hotter stars one or more spectra were of too low $\mathrm{S} / \mathrm{N}$ to provide reliable measurements; the observations being thus incomplete for these stars, we excluded them from subsequent analysis. Therefore, we successfully performed a RV analysis for 145 stars, out of which 41 fall along the EHB.

Our analysis revealed that the errors given by $\mathrm{CC}$ alone were underestimated. We evaluated this effect by analyzing statistically the distribution of $\frac{\Delta(\mathrm{RV})}{\sigma}$ for the cooler stars $\left(T_{\text {eff }} \leq\right.$ $10000 \mathrm{~K})$. Among this subsample, we do not expect a population of very close binaries, and observed variations can be ascribed only to random errors. We found that the latter were underestimated by a factor of 1.44 . We expected this factor to increase for decreasing $\mathrm{S} / \mathrm{N}$, but repeating the analysis in different temperature bins we found only small variations. Moreover, evaluating the correction factor for hotter stars would invalidate our results, because RV variations due to binarity, if not properly identified, would spuriously enlarge the estimated errors and thus incorrectly lead to the lack of detected binaries. Hence we applied the correction by a factor 1.44 to the whole sample, as a good approximation valid for all temperatures.

Our results are summarized in Fig. 1. For each star, we plot the maximum RV variation and the $3 \sigma$ interval. Effective temperatures were estimated using the color- $T_{\text {eff }}$ relation derived in Moni Bidin et al. (2006b). Because each datum is the maximum of six measurements, the probability that each datum exceeds $3 \sigma$ is $1.6 \%$, and we expect 0.66 false detections out of $41 \mathrm{EHB}$ targets. We adopted this threshold to select objects displaying 


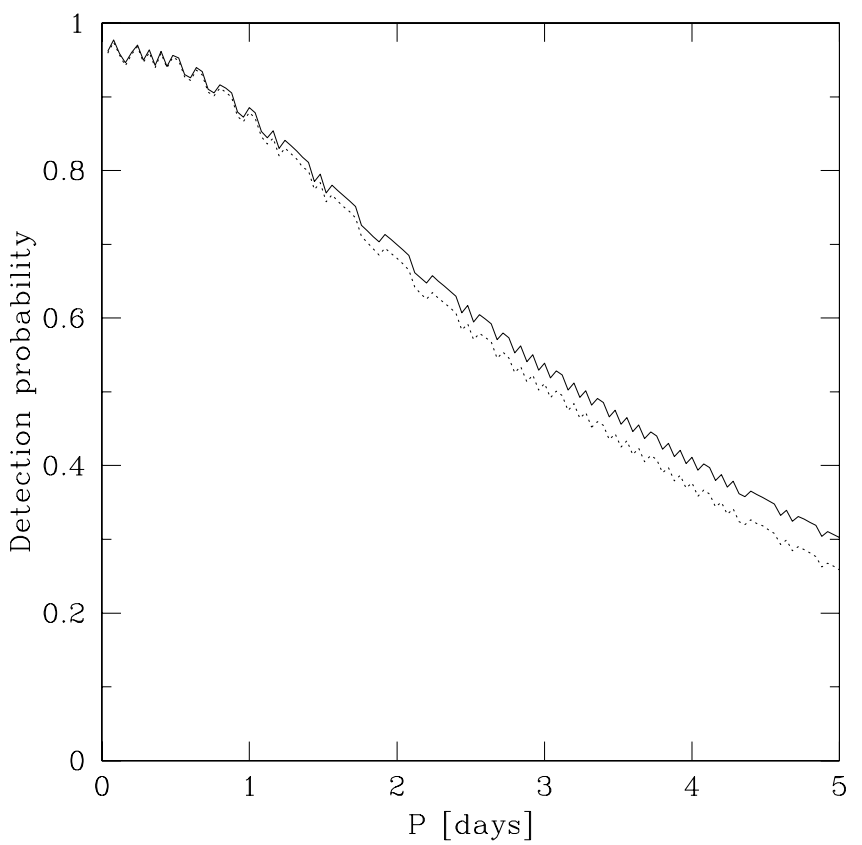

Fig. 2. Probability of detecting a close binary systems in our survey, as a function of its period. As probability varies with both times of observations and errors in the measured RV variations, we plot both the average over the probabilities for each individual star (solid line) and the one calculated assuming a fixed error of $10 \mathrm{~km} \mathrm{~s}^{-1}$ for all stars (dotted line).

suspect variations to be further analyzed, but we found no need to discuss border cases because the only candidate found shows variation at the $10 \sigma$ level $\left(16.1 \pm 1.6 \mathrm{~km} \mathrm{~s}^{-1}\right)$. This star was already highlighted by Moni Bidin et al. (2007b) for its red color and evident $\mathrm{Mg}$ Ib triplet, signatures of a cool MS companion. We are currently analyzing data for this star, which turns out to be the first $\mathrm{sdB}+\mathrm{MS}$ close system ever found in a globular cluster. In summary, we come out with only one good close binary candidate in our whole sample of $145 \mathrm{HB}$ (41 EHB) stars.

In Fig. 2, we plot the estimated detection probability in our survey as a function of the period. This was calculated as in Moni Bidin et al. (2006b), assuming a mass of $0.5 M_{\odot}$ for both components and circular orbits, and integrating over the phase and the orbital inclination angle. We derived the detection probability for each individual star (given sampling times in Table 1 and the aforementioned $3 \sigma$ values), and then averaged it to obtain the final curve. We also checked that other reasonable ways of tackling the problem (for example, assuming a fixed $3 \sigma=10 \mathrm{~km} \mathrm{~s}^{-1}$ for all stars in the sample) did not change our results by more than $1-3 \%$. Clearly, the detection probability rapidly falls down for increasing periods, as a consequence of the observations being restricted to a single night; accordingly, we will limit our analysis to systems with $P \leq 5 \mathrm{~d}$. It must be emphasized that such a limitation is not particularly severe, since many surveys have revealed that the bulk of the field binary sdB population has periods of approximately $P \approx 1 \mathrm{~d}$, systems with $P \geq 5 \mathrm{~d}$ constituting only the tail end of the period distribution (see, for example, Morales-Rueda et al. 2003).

Using the calculated detection probability, we estimated the close binary fraction $f_{P \leq 5 \mathrm{~d}}$ among EHB stars as in Maxted et al. (2001), assuming a flat distribution of periods, and the successful detection of one binary out of 41 targets. We calculated $f_{P \leq 5} \mathrm{~d}$ assuming a Gaussian distribution in $\log P$, as in Maxted et al. (2001) and Napiwotzki et al. (2004), which better represents the period distribution for field sdBs - but the results do not differ noticeably. The derived probability distribution peaks at $f=4 \%$. It is markedly non-Gaussian, but it falls below $5 \%$ for $f \geq 16 \%$. We conclude that the best estimate (i.e., the most likely value) for $f_{P \leq 5 \mathrm{~d}}$ is $4 \%$, and that $f_{P \leq 5 \mathrm{~d}} \leq 16 \%$ at the $95 \%$ confidence level. Our derived upper limit is in perfect agreement with the one that was found for $f_{P \leq 10 \mathrm{~d}}$ by Moni Bidin et al. (2006b). We note that these results are at variance with the preliminary ones previously obtained by Peterson et al. (2002), who claimed the detection of many close binaries among the EHB stars in the same cluster. The reader is referred to Moni Bidin et al. (2006b) for a discussion of this discrepancy.

\section{Is there a close binary fraction-age relation?}

Using an independent dataset, a sample more than twice as large as in Moni Bidin et al. (2006b), and a resolution in RV variations that is higher by almost a factor of two, for the first time we were able to find a good binary candidate among the EHB stars in NGC 6752. That notwithstanding, our results confirm that the corresponding (close) binary fraction $f$ is very small in NGC 6752, with a most likely value of $f=4 \%$ and an upper limit of $f=16 \%$ at the $95 \%$ confidence level.

There are hints that a small $f$ is not a peculiarity of NGC 6752, but could also be a characteristic of other globular clusters (Moni Bidin et al. 2006a). This is in sharp contrast with the situation for field sdB stars, where close binaries are at least a factor of ten more frequent, comprising up to $70 \%$ of the entire sdB population (see Sect. 4 in Catelan 2007, for a recent review). There is however no knowledge about cluster EHB stars in longperiod binaries, or with a close low-mass companion, because no survey has investigated their role yet. These kind of systems are known to exist among field sdBs, but are just a minor population, and their presence in GCs would not alleviate the striking contrast with field results.

What is the origin of this startling difference between field and cluster EHB stars? We believe that it may not be completely unexpected: there should be a relation between the close binary fraction and the mean age of a sdB population, as a consequence of the different efficiency of binary channels responsible for EHB star formation.

Theoretical arguments strongly suggest that sdB stars in close binary systems should have undergone at least one common envelope (CE) phase. Although Han et al. (2002) found an upper limit for the initial mass of the $\mathrm{sdB}$ progenitor in this scenario, they also pointed out that within the permitted values a higher initial mass favors the CE channel, and leads to sdB binaries with shorter periods. In fact, a higher mass implies a more tightly-bound envelope, which requires a greater amount of (orbital) energy to be released. On the other hand, Han et al. (2002) explored the stable Roche Lobe Overflow (RLOF) scenario, and found that a higher initial progenitor mass makes it harder for the RLOF to be stable (see their Table 3), because the minimum mass of the companion increases with increasing progenitor mass. For higher values, fewer MS and white dwarf (WD) secondaries are sufficiently massive for the activation of this channel (sdB's with neutron star companions are indeed very rare). The progeny of systems that underwent stable RLOF, that is wide binaries with very long periods, is therefore generated primarily by progenitors of lower initial mass.

In light of these results, a relation between $f$ and the mean initial mass of the sdB progenitors could be naturally expected, hence implying a relationship between $f$ and (mean) age. More specifically, one may naturally expect that field sdB's formed 
from progenitors with a wide spectrum of initial masses (up to about $2 M_{\odot}$ ), whereas in an old population (such as in globular clusters) only the progeny of less massive stars are currently found on the EHB, those of more massive ones having long evolved away from the He-burning phase. The stable RLOF is an efficient channel for sdB formation in the old case, while the $\mathrm{CE}$ one is not - and the CE itself would be released at earlier stages, before the orbits shrink substantially. Therefore, in old populations we should expect to find predominantly wide binaries, or/and single EHB stars formed through other channels (see Catelan 2007, for a recent discussion). In fact, WD mergers, the third binary channel studied by Han et al. (2002), can form (single) sdB stars, and is expected to be particularly efficient for old populations (see below). Moreover, it may be expected to play an important role within the dense environment of a globular cluster, where stellar encounters can harden close binaries (Heggie 1975) and thus enhance channel efficiency, as proposed earlier by Bailyn \& Iben (1989). However, dynamical effects are not required for the formation of EHB stars (Whitney et al. 1998), as indicated by the lack of radial gradients among the EHB stars in NGC 2808 (Bedin et al. 2000) and $\omega$ Centauri (NGC 5139; D'Cruz et al. 2000).

There are other results in the recent literature that provide support to our proposed scenario. In fact, Napiwotzki et al. (2004) already invoked a possible $f$-age or $f$-metallicity relation to explain their results. Among field sdB stars, they found a close binary fraction lower than in previous surveys, and noted that their sample was on average fainter, possibly including more thick disk or halo (hence older/metal poorer) stars. This suggested difference between the data used has never been fully investigated, and a further check of the populations of these stars would be invaluable. Even stronger support is provided by the recent simulations by Han et al. (2007), modeling the UV upturn of elliptical galaxies from a binary sdB population. Their Fig. 7, though aimed at reproducing the spectral energy distribution of elliptical galaxies, shows how the relative contribution of sdB's formed through different channels evolves with time. One finds that the contribution to the UV flux from RLOF sdB's is essentially constant with time, whereas the one from sdB's that experienced the CE phase first becomes important for a population of age $1.5 \mathrm{Gyr}$ and then slowly fades, being more and more marginal for increasing ages. On the other hand, for populations older than 5 Gyr these authors find that sdB's formed through the merger channel dominate - but these are not binaries any longer. We suggest that these theoretical results implicitly hint at a solution to the heretofore puzzling lack of close EHB binaries in globular clusters, on the one hand, and their large numbers among field stars, on the other.

As an alternative to the binary scenario, it is worth noting that many single-star evolutionary channels have been invoked to explain EHB star formation in GCs, including interactions with a close planet (Soker 1998, see also Silvotti et al. 2007), He mixing driven by internal rotation (Sweigart \& Mengel 1979; Sweigart 1997) or by stellar encounters (Suda et al. 2007), dredge-up induced by H-shell instabilities (von Rudloff et al. 1988, but see also Denissenkov \& VandenBerg 2003), close encounters with a central, intermediate-mass black hole (Miocchi 2007), and a sub-population of stars with high helium abundance (e.g., D'Antona et al. 2005).

We note that any EHB formation mechanism involving single progenitors should be particularly inefficient among field stars (but possibly not so among GCs), since the EHB progenitors are on average younger, and thus more massive, in the field than in GCs. As a consequence, a much higher amount of RGB mass loss is needed to successfully produce a EHB star in the case of a field progenitor, whereas the ancient GC red giants have a much smaller envelope to be removed before they become EHB stars. This notwithstanding, a small component of single-star progeny may still be needed among field stars to fully explain the available observations (Lisker et al. 2005).

Acknowledgements. We warmly thank Yazan Momany for providing us with his accurate astrometric data. M.C. is grateful to Allen V. Sweigart for insightful discussions on the origin of hot HB stars. C.M.B. acknowledges Universidad de Chile graduate fellowship support from programs MECE Educación Superior UCH0118 and Fundación Andes C-13798. Support for M.C. is provided by Proyecto Fondecyt Regular \#1071002. M.A. was funded by FONDAP 15010003.

\section{References}

Allard, F., Wesemael, F., Fontaine, G., Bergeron, P., \& Lamontagne, R. 1994, AJ, 107, 1565

Aznar Cuadrado, R., \& Jeffery, C. S. 2001, A\&A, 368, 994

Bailyn, C. D., \& Iben, I. J. 1989, ApJ, 347, L21

Bedin, L. R., Piotto, G., Zoccali, M., et al. 2000, A\&A, 363, 159

Catelan, M. 2007, [arXiv:0708.2445]

D’Antona, F., Bellazzini, M., Caloi, V., et al. 2005, ApJ, 631, 868

D'Cruz, N. L., O'Connell, R. W., Rood, R. T., et al. 2000, ApJ, 530, 352

Denissenkov, P. A., \& VandenBerg, D. A. 2003, ApJ, 598, 1246

Ferguson, D. H., Green, R. F., \& Liebert, J. 1984, ApJ, 287, 320

Han, Z., Podsiadlowski, P., Maxted, P. F. L., Marsh, T. R., \& Ivanova, N. 2002, MNRAS, 336, 449

Han, Z., Podsiadlowski, P., Maxted, P. F. L., \& Marsh, T. R. 2003, MNRAS, 341, 669

Han, Z., Podsiadlowski, P., \& Lynas-Gray, A. E. 2007, MNRAS, 380, 1098

Heber, U., Moehler, S., Napiwotzki, R., Thejll, P., \& Green, E. M. 2002, A\&A, 383,938

Heggie, D. C. 1975, MNRAS, 173, 729

Lisker, T., Heber, U., Napiwotzki, R., et al. 2005, A\&A, 430, 223

Maxted, P. F. L., Heber, U., Marsh, T. R., \& North, R. C. 2001, MNRAS, 326, 1391

Menzies, J. W., \& Marang, F. 1986, in Instrumentation and Research Programmes for Small Telescopes, IAU Symp., 118, 305

Miocchi, P. 2007, MNRAS, 381, 103

Momany, Y., Piotto, G., Recio-Blanco, A., et al. 2002, ApJ, 576, L65

Moni Bidin, C., Moehler, S., Piotto, G., et al. 2006a, [arXiv:astro-ph/0606035]

Moni Bidin, C., Moehler, S., Piotto, G., et al. 2006b, A\&A, 451, 499

Moni Bidin, C., Catelan, M., Villanova, S., et al. 2007a, [arXiv:0711.0506]

Moni Bidin, C., Moehler, S., Piotto, G., Momany, Y., \& Recio-Blanco, A. 2007b, A\&A, 474, 505

Moran, C., Maxted, P., Marsh, T. R., Saffer, R. A., \& Livio, M. 1999, MNRAS, 304,535

Morales-Rueda, L., Maxted, P. F. L., Marsh, T. R., North, R. C., \& Heber, U. 2003, MNRAS, 338, 752

Morales-Rueda, L., Maxted, P. F. L., \& Marsh, T. R. 2004, Ap\&SS, 291, 299

Morales-Rueda, L., Maxted, P. F. L., Marsh, T. R., Kilkenny, D., \& O’Donoghue, D. 2006, Baltic Astronomy, 15, 187

Munari, U., Sordo, R., Castelli, F., \& Zwitter, T. 2005, A\&A, 442, 1127

Napiwotzki, R., Karl, C. A., Lisker, T., et al. 2004, Ap\&SS, 291, 321

Peterson, R. C., Green, E. M., Rood, R. T., Crocker, D. A., \& Kraft, R. P. 2002, ASP Conf. Ser., 265, 255

Reed, M. D., \& Stiening, R. 2004, PASP, 116, 506

Saffer, R. A., Livio, M., \& Yungelson, L. R. 1998, ApJ, 502, 394

Silvotti, R., Schuh, S., Janulis, R., et al. 2007, Nature, 449, 189

Soker, N. 1998, AJ, 116, 1308

Suda, T., Tsujimoto, T., Shigeyama, T., \& Fujimoto, M. Y. 2007, ApJ, 671, L129

Sweigart, A. V. 1997, ApJ, 474, L23

Sweigart, A. V., \& Mengel, J. G. 1979, ApJ, 229, 624

Tonry, J., \& Davis, M. 1979, AJ, 84, 1511

Ulla, A., \& Thejll, P. 1998, A\&AS, 132, 1

von Rudloff, I. R., Vandenberg, D. A., \& Hartwick, F. D. A. 1988, ApJ, 324, 840

Whitney, J. H., Rood, R. T., O'Connell, R. W., et al. 1998, ApJ, 495, 284

Williams, T., McGraw, J. T., Mason, P. A., \& Grashuis, R. 2001, PASP, 113, 944 